\title{
EXTRACTION OF PRIMITIVES AND OBJECTS FROM HSHAPES
}

\author{
C.Bolognesi ${ }^{1}$, V.Caffi ${ }^{1}$ \\ ${ }^{1}$ ABC Dep. Politecnico of Milano, Milano, Italy, (cecilia.bolognesi, vittorio.caffi)@polimi.it
}

\section{Commission II}

KEY WORDS: point clouds, shapes, vaults, BIM, lines

\begin{abstract}
:
This paper deals with 3D morphological studies of architecture surveyed with TLS to allow the modelling of a Renaissance villa in a BIM environment; the aim is to create Renaissance objects to be shaped into a parametric software and made recognizable in a BIM environment balancing level of accuracy and shortness of workflow. The approach of this study lies in the comparison of different workflows, using different BIM software and apps, useful to extract transverse ridge or primitive ribs from the point cloud of walls as vaulted ceilings, shape accurate objects, considering their level of detail and their morphological and typological aspect. The first steps of Data Processing from 3D Survey started with an accurate cleaning and transformation in appropriate but different format to be analysed into different environment and treated for a modelling phase. The different results of the different workflow have been compared to measure grade of simplification, when existent, of the single objects compared. The case study deepens the entrance of a Renaissance Villa in Tuscany, Villa di Poggio a Caiano, in its main elevation considering the vaults of porches and entrances as barrel vaults or lunette-like with double-curved nails.
\end{abstract}

\section{INTRODUCTION}

Awareness of the benefit of BIM modelling is now a fact as the benefit of 3D digital technologies for survey in the heritage field (Fassi et al., 2016). But the introduction of the paradigm of complexity own by cultural heritage and the necessity to maintain its richness in the following derived BIM model needs new processes and new approaches (Fay, 2011). Difficulties of modelling the existing architectures or more, the ancient ones, in a BIM environment remains a time and almost point of weakness of the whole HBIM process. Post processing of data set as well as degrees of accuracy obtainable only with the operator ability, often with skills derived from information technology, computational skills, interoperability among software weaken the usability of the system in spite of an ever-increasing demand of the researchers and public administrator especially in European countries. Meantime the possibility of using apps facilitating the extraction of lines-objects from the point cloud and the possibility of using shorter workflows grows trying to satisfy a necessity (Wang et al.,2015).

\subsection{State of the art}

In this perspective investment of research and industry proceed forward for new tools that are more and more automated. In terms of investments, therefore, the whole sector is moving into applications that favour the inclusion of the point cloud in the software to define useful and possibly automated tools and procedures for its interpretation. Very often additional tools are required to bring the existing point cloud into environment that allow to structure 3D object derived from the point cloud. Shape modelling end extraction of specific morphologies in any digital workflow are now ready to include in this topic the significant passage from line drawing to volume drawing and "object" creating. Working in a BIM environment often requires a pipeline with different tracks mainly manually managed and measured as extraction from the point cloud; this path generally starts from the shaping of the line by the operator after slice extraction from the point cloud, horizontal, vertical or both in a dense mixture to define the morphology of the shape (Banfi, 2016). Working in a BIM environment can involve volume subtraction directly in the modeler to define surface complexity, or mass modelling
(Bowles, 2013) Beside this path geometrical algorithms have reached a specific capability to portray complexity of surfaces, leaving sets of information related to the object and interoperability problems to be solved in a second time.

\section{RELATED WORKS}

As related works show us, every modelling could lead to data loss (Murphy, 2009); defining geometric characteristics generally needs a lot of generative practicing, software skills, interdisciplinary groups also.

Literature and researches on the subject offers several scientific papers interpreting and going deep into complexity often in a single specific section without comparing different workflows. Since the possibility to develop BIM models starting from point clouds has become more real, the need for semantic research of the objects that can be assumed in a BIM environments emerged. But if TLS and photogrammetric surveying technologies have grown over the last ten years so much (Murphy, 2013) as to give output of complex and dense point clouds with strict precision, generation of BIM objects from point cloud is still the time consuming task. Geometry derived from point clouds has to be dividend in semantic objects, turned into different and

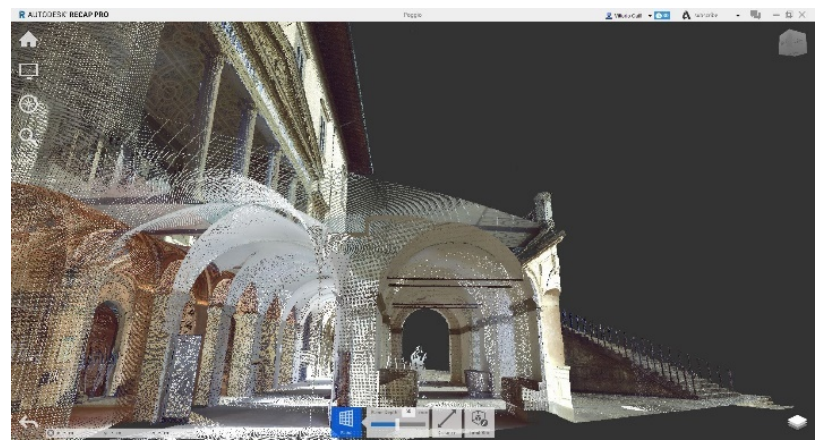

Figure 1: post processing of the point cloud of the entrances of the villa in Recap. 
specialized models, assuming different data sets related to the different uses they are designated. In recent times both the UE (UE, 2015) and UK Governments (Historic England, 2017) indicated laser scanner technology as the most suitable method for data capture. However, this task has not been followed by any thought about how to integrate it into the BIM environment; the cause of it can be related to the development of the information connected to LoD definition in general and particularly to the discussion related to the LoD of a point cloud. (Thomson et al, 2015). The actual main proposal deals with the possibility of the point cloud as a LoD 0 with the possibility to host or generate higher level of details.

Main researches regarding BIM models derived from point clouds surely starts from Murphy first scientific writings till the most recent ones (Murphy et al 2009; Murphy et al 2011; Murphy et al 2014) becoming a reference for many different works (De Luca et al 2011) followed by other authors by Steven Fay in his fundamental work regarding Ottawa Parliament (Fai 2014).

Considering the point Cloud as a 0 Lod the geometric theme, the task to define lines surfaces and volumes for the BIM model, remains. A first simplification of the theme through last researches can be organized in three different main workflow related only to geometric modelling of the point cloud.

A first track concerns the automatic recognition of semantic objects within the cloud. This line of research started at least 25 years ago (Nagel et al. 2009) and it is still ongoing far to be solved. Most recent researches deals with automatic recognition of specific 3D shapes from point clouds of cultural heritage assets supported by applied tailored tools using PLY, PTX and PTS formats as input files.

To make this tool functional and widely used, (Cembranos et al. 2018) it has been currently developed also as a plug-in for Revit BIM software as experimental tool. This is the case of a development grown in Inception research for Horizon but as this is an experimental software it has not been tested for this work. This last frontier deals with automated algorithms strictly connected to parametric logics processing large quantity of data to be able to detect segments. This plug-in for REVIT is programmed in $\mathrm{C}++$ and supported by the Point Cloud Library (PCL) as standalone, large scale, open software project for image and point cloud processing; the tool is said to be able to precisely display the detected features in a unique working project (RVT/RFA). The Point Cloud Library through his algorithms detect geometrical primitives such as planes, cylinders, spheres, cones or torus. The vast majority

of the architectural geometries making up the built heritage can be modeled by means of these primitives. Theoretically, a point cloud can be completely processed with the proposed library.

A second track concerns the manual workflow with definition of lines or anyway clear generative linear geometries in the cloud, not yet referable to a process of semantic reduction of BIM objects, but useful to define it. The approach starts at the beginning of the second decade of 2000 with several research lines among surveyor practicing laser scanner and photogrammetry, in a first phase at the beginning of BIM modelling, generally more focused on the point cloud than the BIM model. (Umili, et al 2011), using mainly available software. The development of this research line has grown through several papers (Garagnani, 2012) and different technicalities referred to different areas of application of the survey of the existing (De Luca, 2012), (Barazzetti 2015).

The third track concerns a semi-automated workflow where the features of parametric objects can be modified within the model using different software and/or plug-in as described and tested in the following chapter (Dore, 2014).

\section{DEVELOPED METHODOLOGY AND WORKFLOWS}

This paper proposes different workflows where the point cloud of a Renaissance Villa with some spaces enriched with vaulted ceilings is managed from the $3 \mathrm{D}$ survey to the creation of generative elements like lines, nurbs, and mesh; to do so different authoring software are compared to obtain manually or with a semi-automated workflow shape, nurbs and objects also to be integrated with parametric applications in different BIM environments.

\subsection{A semi-automated workflow from the cloud to BIM- Revit}

As we already know BIM environment is formed by 3D objects related one to the other; the objects are a geometry associated to information while pure modelling can create meshes and nurbs only. Solids with a good level of correspondence to the existing but with no information or parametrization are useless in a BIM environment.

The process from nurbs derived from Point Cloud to object is easily summarized in: laser scanning generation, Photogrammetry application, cloud registration, cloud cleaning, extraction of primitives with automated/semi-automated/manual modelling procedures, nurbs interpolation for generation of complex surfaces traced from points or lines extruded and interpolated, definition of LoD to be reached, integration of nurbs into BIM environment, parametrization, associating information. A typical semi-automated path to import the point cloud in Revit is: preparation of the cloud in Recap: convert the file to .Rcp or . .Rcs for Autocad when proceeding with slicing, or .Pts format for Rhino (Revit reads only proprietary format Rcp/RCS) using Recap. If the version of Recap in use is the full one, it is possible to clean what is not necessary and clip the image to understand the model better. Recap is useful as we can use it as an Autocad app and visualize the single clouds after their registration in .Rcs.

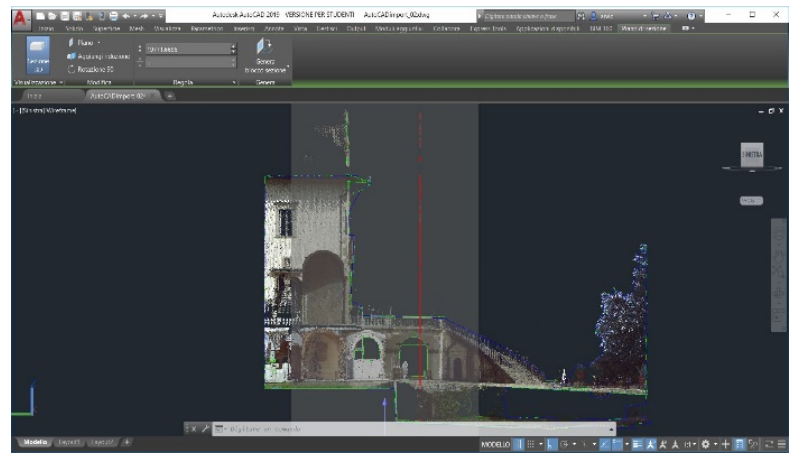

Figure 2: line generation in Autocad

Orto and other views as perspective allow a sequence of commands such as offset (within some centimeters), select, crop, to outline primitives and shape slices and then create groups. It is mandatory to draw with snap setting off or useful setting. After association with appropriate layers we can import into Revit primitives obtained and start working with parameters to be associated. Passage through Autocad before Revit allows defining with better precision defining snap with $2 \mathrm{D}$ or $3 \mathrm{D}$ options, slice determination and automatic extractions of lines. For more complex shapes a solution is provided with Rhino: processing the cloud with Recap and saving it with. Pts format allows to outline new primitives with Rhino displaying other options and morphological precision. The profiles of the vault can be traced; we consider using controls in different points to be 
checked along edges and in the cloud. Different edges can be joined to define the vault and a comparison can be done between the result and the point cloud. Autocad catch Rhino files and allows export from Rhino to Revit. Revit modelling if precise can become time consuming and not always comfortable. In Revit Geometry can be added of parameters and information becoming parametric objects.

We could Import the point cloud directly in Revit also, with a simplified but less specific workflow, mainly useful for survey of recent buildings, with simplified geometries. The typical workflow is insert the file RCP in Revit; adjust position and set main levels re-orienting the model; set a reference view (front) using a wall and elevation marker; sketch using the point cloud as a reference; then insert an elevation marker, it will point automatically to the wall, then it'll be possible to set other three main views back/left/right; re-orient the model to the four main views; crop the views (plans, elevations, 3D) in order to limit the visible to the needed parts.

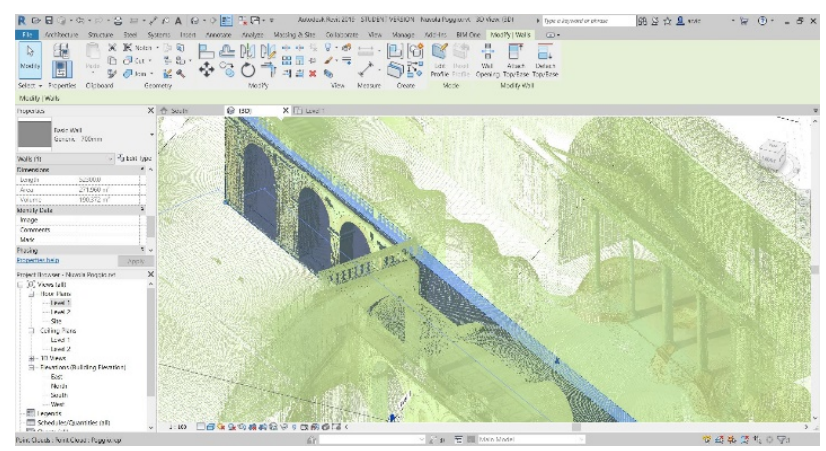

Figure 3: wall inserted in Revit

Regular elements - slabs, stairs, orthogonal walls can be easy modelled using the regular Revit tools. If modelling components it is necessary to use the right category, if modelling masses, it is later generally possible to generate components from surfaces. Not regular elements or complex curves and intersections, not necessary spline, have to be modelled using specific strategies to be chosen according to each element. as described before and can't follow this direct import.

\subsection{A semi-automated workflow from the cloud to BIM-Revit through Point-Cab}

The workflow here illustrated uses a German software, Point-Cab to better manage point clouds and allow a semi-automated workflow. Semiautomatic workflow considers to use Point-Cab to:

1.Organise the model - Point-Cab for Revit (plug in) helps to automatically create Levels as from the .Pcp file created by Point$\mathrm{Cab}$

2. Generate components- Point-Cab for Revit (plug in) helps to create walls and insert components such as windows and openings. Walls are marked in Point-Cab. The connection PointCab Revit allows to generate walls that are proper Revit families and Windows, doors and openings also.

3. Generate and import profiles from other software.

a. Point-Cab can set cross section, elaborate section (generate image), elaborate vector file dwg and/or dxf; vector files can be imported in Revit and use to model with the ordinary 3D tools (families and/or masses/components in place).

Assumed Point-Cab to manage clouds gives excellent results in extracting edges from the large part of the elements of the case history of the Villa here developed but less results in extracting edges from vaults, still revealing time consuming and difficult task.

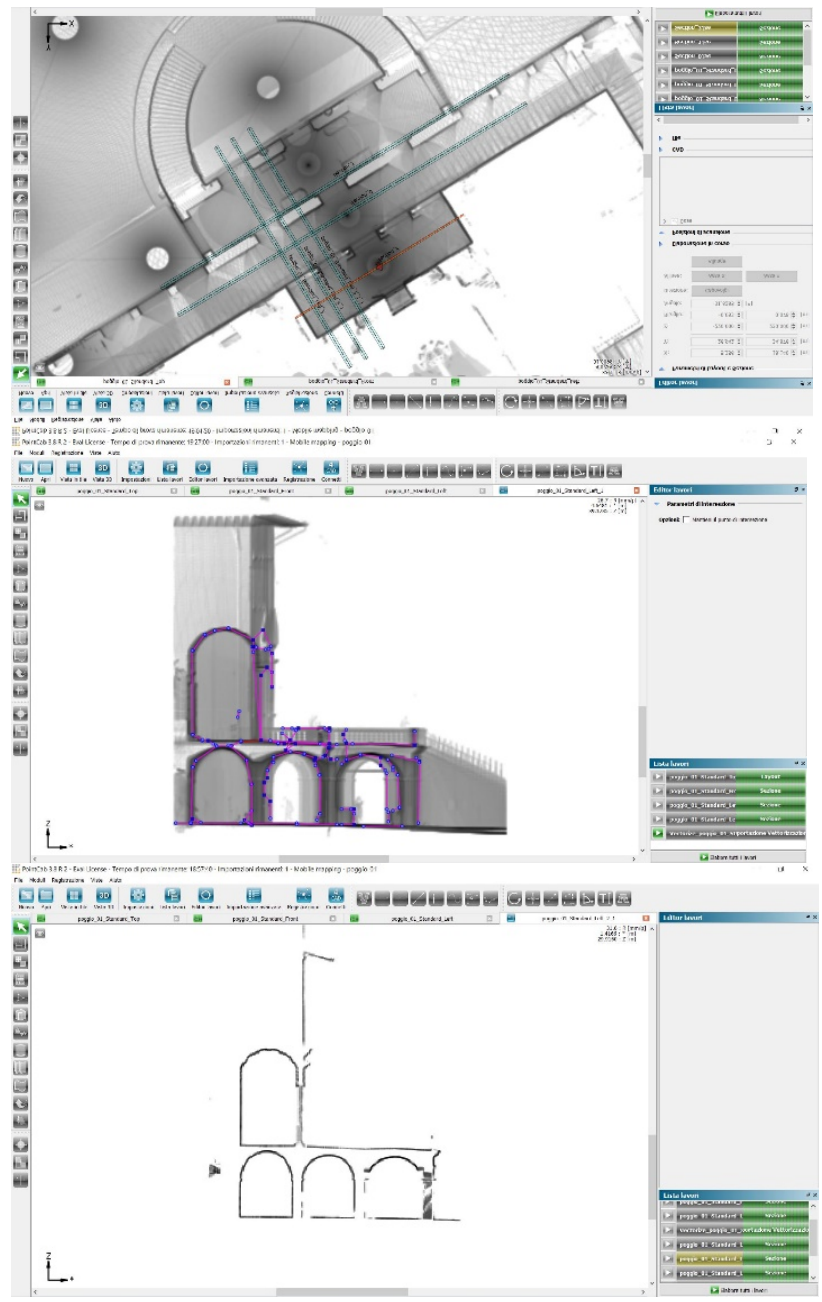

Figure 4: a part of the workflow in Point-cab for revit: section positioning; section outlined; lines extracted.

\subsection{A semi automated workflow from the cloud through Rhino-Grasshopper Point-Cab to BIM-Archicad.}

The most common problem in Scan to BIM processes is paradoxically the excess of the points that compose the point cloud, the factor that generally determines the quality of the survey. This is the reason why it is extremely useful to have a system able to manage the amount of points scattered in the space for which Point-Cab was used autonomously, exporting the entities generated in Archicad; Point-Cab has its own app to exchange files directly.

The point cloud point was exported from Recap in a .e57 file. A not very high density of points was detected, that caused different parts to be not really dense: inside the loggias the TLS should be positioned between pillar and pillar to have a complete and precise representation and the size of the loggias did not allow this full detail.

Point-Cab automatically generates three views, from which it is possible to generate Orto photos, correctly geo-referenced with respect to the point cloud that originate them; it is possible to visualize and work with how many Orto photo views are needed, plants, elevations and sections. 
The transformation of Orto photos into vector allows to automatically obtain files in the formats .dwg, .dxf or .dae.

All the generated views, whether they are raster Orto photos or vector files, can be imported into the modelling software; in this case Archicad confirm them exactly in their original position, referring to the precise position from which they were derived from the point cloud keeping them available from the same plants, elevations and sections as well as from the $3 \mathrm{D}$ window. They therefore automatically form a more or less dense network of horizontal and vertical section lines, which will constitute the backbone where to structure our BIM model.
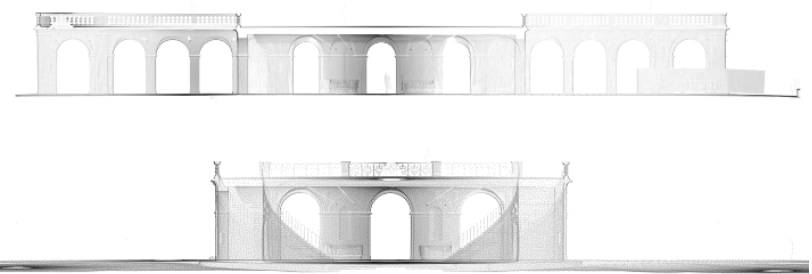

Figure 5: two section visualized in Point-Cab

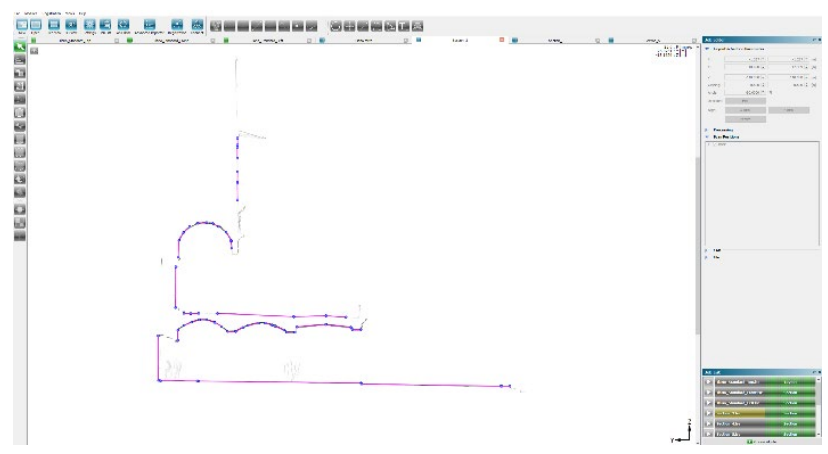

Figure 6: a vector section visualized in Point-Cab

When imported the point cloud in the .e57 file (but the .xyz format is also available) it matches with the previously embedded views.

The 3D sections and the cloud of points in Archicad correspond precisely: the sections, both vertical and horizontal, are used as a base for the development of walls, slabs and vaults; the points cloud is especially useful as a check tool throughout the modelling process and subsequent recovery and management of the existing one.

The point cloud is available in each phase to evaluate the consistency and to quantify the delta between virtual and real.

Another feature of the Point-Cab / Archicad plug-in is the ability to model native Archicad objects directly from the point cloud in panoramic mode.

Archicad tools dos not model the geometry of the vaults, lunettelike with double-curved nails, shapes for which Grasshopper, Rhinoceros's algorithmic programming tool, was used.

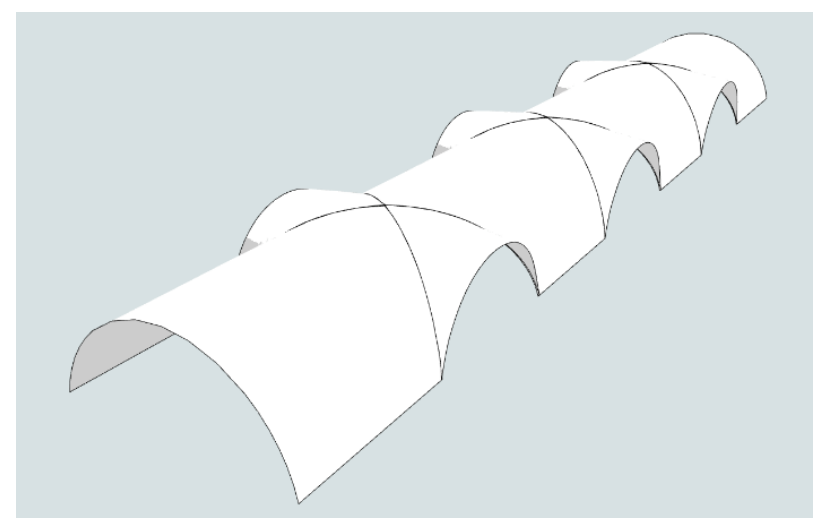

Figure 6: the lunette-like with double-curved nails

The barrel vault has been placed in Archicad taking as reference vector section by Point-Cab extruded along its length on the plant generated by Point-Cab. Bases of lateral lunettes correspond to the arched openings in the walls

Grasshopper acquires the entities of Archicad and breaks them down into its constituent elements: the extrusion of the generating vault and the arches of the lunettes. After that the various geometrical operations are elaborated in terms of projection of the base lines on the topological plan of the barrel vault.

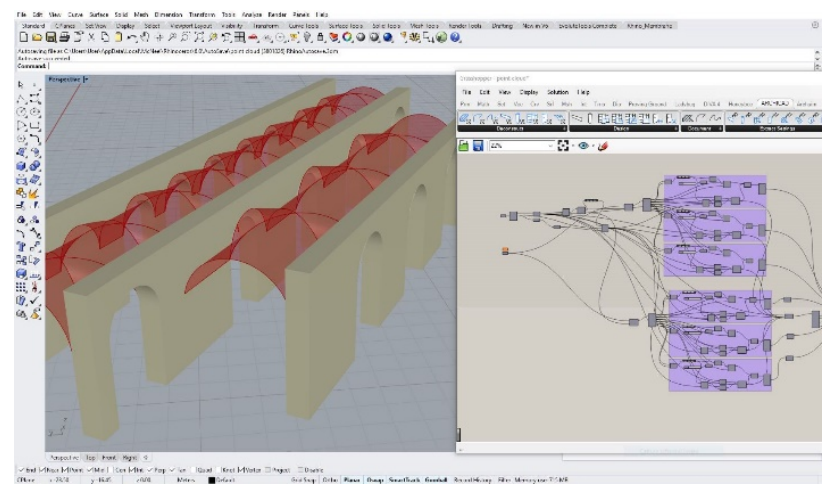

Figure 7: Grasshopper interface for morphology modelling of the vault.

The advantages provided by this methodology are the speed and accuracy but above all the fact that, once the algorithms have been defined starting from the existing reference geometries, the fact that the final result can be modified from the starting geometries themselves, without having to reorganize the algorithmic structure:

If we modify, for example, the height of the vault or the set of lateral openings in Archicad (taking the point cloud as a reference), the whole system of the roof changes following the new height.

We have therefore established a direct correspondence between the survey model and the BIM model which is very useful for the management of primary geometries.

Instead of using a vault generated by a simple linear extrusion, we can plan to shape geometry resulting from the interpolation of several section lines, so as to have a result more relevant to the physical reality of the object, where the same principle is applicable to each element of the model. 


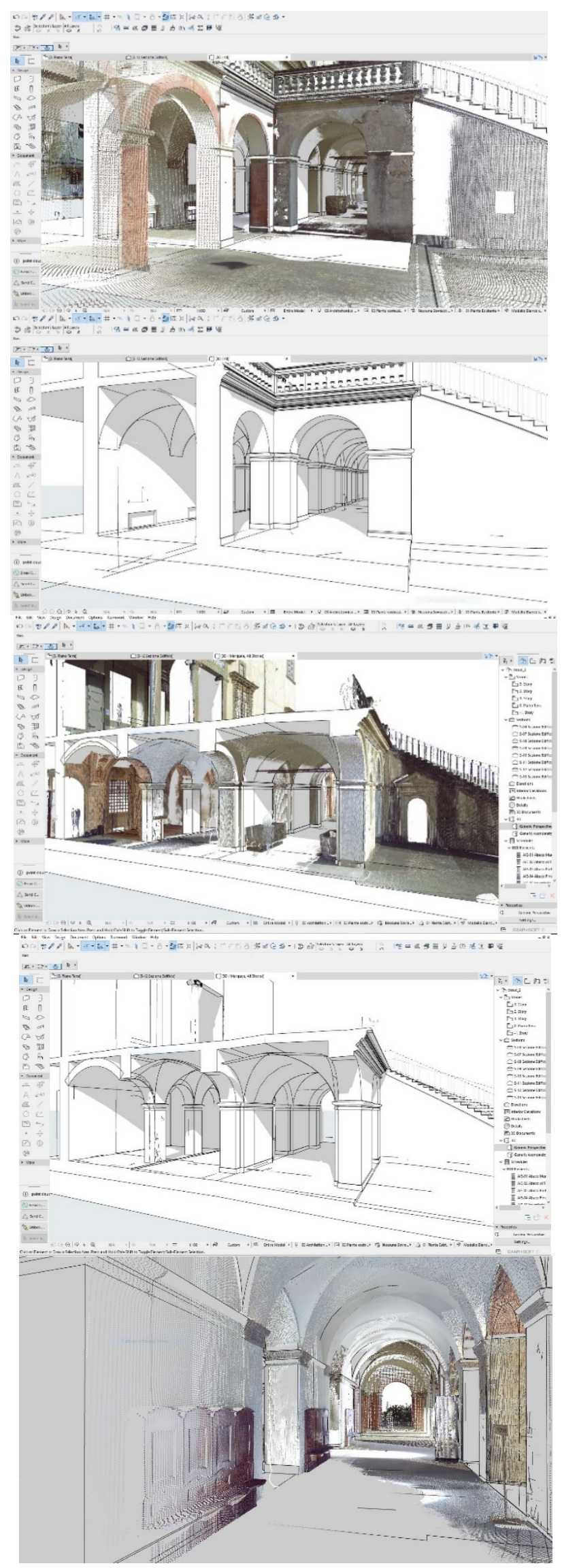

\section{RESULTS}

Laser scanner technology is the first tool for modelling existing architectures and is now essential, like photogrammetry, for any 3D modelling based on credible survey. The workflows presented here explores some possibility of semi-automatic modelling of clouds in BIM environment with Revit or Archicad software using support passing through Autocad, Rhino, Point$\mathrm{Cab}$ and Grashopper environments.

Autocad has been used to find edges to be imported into Revit or as a transit to transmit Rhino files to Revit.

Grasshopper has been used as Rhinoceros's algorithmic programming tool. The advantages deriving from designing profiles in Autocad or Rhino are the precision of the line derived from slicing then imported to elaborate Revit procedures. The advantages of Point Cab are greater manageability of views and better automation in importing set of sections in BIM software. However, not all the captures of the edges were easily automated in Point-Cab especially the vaulted spaces, perhaps due to the decimation of the cloud coming from the laser survey also; Point Cap reveales its difficulty for complex surfaces.

Grasshopper has proved to be useful a useful software for parameterization of objects shaping also in addition of Point $\mathrm{Cab}$ extraction but currently these workflows is suitable for Archicad only.

\section{CONCLUSIONS}

The work carried out here deepens some workflows from point cloud to generation of BIM models using different software, tools and thinking of different uses of the model itself.

This process generally known as a Scan to Bim process seems to be still far from a unique workflows and every time requires defining the model in order to make it efficient for different uses. In spite of different utilization of the model that can be solved embedding informative attributes in the $\mathrm{db}$ every possible simplification of geometry has limits due to the need to satisfy different necessity of accuracy. Point $\mathrm{Cab}$ helps transferring edge lines automated extract from the point cloud into different software (Autocad, Archicad, Revit, Sketch up also) helping Bim object modelling; it would be useful to help the process for every element provided in BIM libraries to complete the whole process helping accuracy and automation in the meanwhile.

\section{ACKNOWLEDGEMENTS}

The authors would like to acknowledge students: Bovi M., Ferraretti E., Pennacchio G., Yuri S for supporting data set collection.

Simone Garagnani for supporting point cloud questions.

Hilario Bourg, Graphisoft, for supporting Archicad questions.

Martin Graner pointcab-software.com

Ralph Schwaiger bim-measurement.com

Andreea Burcescu pointcab-software.com

for supporting Point-Cab question.

The research is part of a general agreement drawn up between the faculty of Histoire de l'art de la Renaissance École Pratique des Hautes Études - Sorbonne, with Sabine Frommel, and AUIC School of Architecture Urban Planning Construction Engineering at the Politecnico of Milano with Cecilia Bolognesi, deduced from a previous agreement between Sorbonne with Alma Mater Bologna.

Figure 8: final model of the entrance ceilings and section vaulted modelling Point-cab, Grasshopper, Archicad 


\section{REFERENCES}

Banfi, F.,2016. Building Information Modelling - A Novel Parametric Modeling Approach Based on 3D Surveys of Historic Architecture. EuroMed 2016, Part I, LNCS 10058, 116-127.

Bowles, J., 2013 Modeling Complex Shapes in Autodesk $\AA$ Revit@ Structure Using the Conceptual Massing Environment, http://help.autodesk.com.s3.amazonaws.com/sfdcarticles/kA230 000000ticNCAQ/handout 1511_SE1511-L-Modeling.pdf (Last accessed on August 2018)

Barazzetti, L., 2016. Parametric as-built model generation of complex shapes from point clouds. In: Advanced Engineering Informatics 30, pp. 298-311

Cembranos, J.R., Fernández, L., Lerones, P., Bermejo, J., Zalama, E., Ioannides, M., 2018. Supporting the Automatic Extraction of HBIM Elements from Point Clouds. EuroMed LNCS 11197, pp. 3-10.

De Luca, L., 2012. Methods, Formalisms and Tools for the Semantic-Based Surveying and Representation of Architectural Heritage. In: Applied Geomatics, no. 1866-9298, pp. 1-25.

Dore, C., 2014. Semi automated generation of as-built façade geometry. Electronic Journal of Information Technology in Construction ITcon Vol. 19, pg. 20-46

European Commission: Getting cultural heritage to work for Europe. Report of the Horizon 2020

Expert Group on Cultural Heritage (2015). https://ec.europa.eu/programmes/horizon2020/en/news/gettingcultural-heritage-work-europe. Accessed November 2018

Fai, K. Graham, T. Duckworth, N Wood, R. Attar, 2011. Building information modelling and heritage documentation. 18th International Conference on Virtual Systems and Multimedia. XXIII CIPA Symposium - Prague, Czech Republic.

Fai, S., Rafeiro,j., 2014. Establishing an appropriate level of detail $(\mathrm{LoD})$ for a building information model (BIM) - West Block, Parliament Hill, Ottawa, Canada, In: ISPRS

Ann. Photogramm. Rem. Sens. Spatial Inform. Sci. II-5 123-130.

Garagnani, S., 2012, Building Information Modeling semantico e rilievi ad alta risoluzione di siti appartenenti al Patrimonio Culturale. In Disegnarecon, Vol. 5, n. 10, Numero Speciale DOCO.

Historic England: BIM for Heritage: Developing a Historic Building Information Model.

Swindon, Historic England (2017). https://historicengland.org.uk/images-books/publications/ bim-for-heritage. Accessed November 2018

Murphy, M., McGovern, E., Pavia, S., 2009. Historic building information modelling (HBIM), Struct. Surv. 27 (4) 311-327

Murphy, M., McGovern, E., Pavia, S., 2011. Historic building information modelling - adding intelligence to laser and image based surveys of European classical architecture, In: ISPRS J. Photogramm. Rem. Sens. 76 89-102.

Murphy, M., Dore, C.,Mc Carthy, S., 2015. Structural simulation and conservation analysis- historic building information model.
Int. Arch. Photogramm. Rem. Sens. Spatial Inform. Sci. XL-5/W4 (2015) 351-357

Nagel, C., Stadler, A., Kolbe, T.H., 2009. Conceptual requirements for the automatic reconstruction of building information models from uninterpreted 3D models. In: T.H. Kolbe, H. Zhang, S. Zlatanova (Eds.), GeoWeb 2009 Academic Track-Cityscapes, vol. 38, ISPRS, Vancouver, BC, Canada, pp. $46-53$.

Thomson, C., Boehm, J., 2015. Automatic geometry generation from point clouds for BIM, Rem. Sens. 7 (9) 11753-11775.

Tommasi, C., Achille, C., Fassi, F., 2016. From point cloud to BIM: a modelling challenge in the cultural heritage field. Int. Arch. Photogramm. Remote. Sens. Spat. Inf. Sci. 41, B5

Umili, G., Forlani, G., 2011. Fusione di DSM ed immagini per l'estrazione automatica di linee di rottura dal DSM. In Atti $15 a$ Conferenza Nazionale ASITA - Reggia di Colorno 15-18

Zolanvari, I., Laefer, S., Debra, 2016. Slicing Method for curved façade and window extraction from point clouds. In:

ISPRS Journal of Photogrammetry and Remote Sensing, Vol. 119 , pp. $334-346$

Wang, C., Cho, Y.K., Kim, C., 2015. Automatic BIM component extraction from point clouds of existing buildings for sustainability applications. Autom. Constr. 56,1-13 (2015) 